\title{
A new two stage adaptive nonparametric test for paired differences
}

\author{
Weiwen MiaO* And Joseph Gastwirth ${ }^{\dagger}$
}

This paper proposes a new adaptive procedure for analyzing paired data. The procedure uses a function of ordered absolute values of the differences to measure tail heaviness of the underlying distribution. The value of the measure is then used to choose an appropriate signed rank test. The new adaptive procedure is shown to preserve the size of the test at its nominal level for all continuous distributions and typically has nearly the same power as the best signed rank test for a wide range of distributions.

KEYWORDS AND PHRASES: adaptive paired test, signed rank test, tail-heaviness.

\section{MOTIVATION AND INTRODUCTION}

Paired data arises in a wide variety of applications (e.g. Sprent and Smeeton, 2001). In a recent security law case where both authors were statistical consultants to the defendant lawyers, a Wall Street firm was charged of favoring a few customers who "shared profits" with them. Since the "profit sharing" funds usually sold their shares through a different firm than the one they acquired them, the regulators need to estimated the profit. Unfortunately, two authorities, the SEC (Securities and Exchange Commission) and the NASD (National Association of Securities Dealers) used different methods to estimate the profits customers made. A natural question is whether the two estimates of profits are essentially the same. As a firm could be examined by each regulator and be accused of "profit sharing" by one regulator and exculpated by the other. Since the two different measurements are for the same stock, the data is naturally paired. Data of 157 IPOs (Initial Public Offering) during an 18-month period were made available to us. Our analysis shows that the differences have heavier tail than the normal distribution. It's better to use the $t_{2}$ or Cauchy scores test to detect the difference.

Paired data also arise in environmental studies where duplicate observations are taken. In 2003, the Michigan Department of Environmental Quality Remediation and Redevelopment Division conducted off-site samplings for the Detroit lead assessment project. Twenty-four soil samples

* Corresponding author.

${ }^{\dagger}$ The research of Professor Gastwirth was supported in part by NSF grant SES-0317956. were collected for lead analysis in vicinity of the former Great Lakes Smelting Company in Detroit, Michigan. These 24 samples are from 12 different locations near the plant with two samples from each location. Our interest is to test whether the two measurements from the same location are the same. Analysis shows that the differences are not normal. (The p-value of the Shapiro-Wilk test is 0.029.) Hence, the paired t-test is not appropriate for the analysis of this data.

Motivated by these two examples, we develop an adaptive procedure that uses a preliminary test to first analyze the tail-heaviness of the underlying distribution of the differences. That information is then used to choose an appropriate signed rank test to analyze the paired differences. For the sample of 157 estimated profits, our adaptive procedure chooses the $t_{2}$ scores test which yields a p-value of 0.009 , about one-third of the one obtained from the paired t-test $(\mathrm{p}$-value $=0.027)$. For the environmental data, the new adaptive procedure also chooses the $t_{2}$ scores test which yields a p-value of 0.038 , showing that the two measurements are actually significantly different. For comparison purposes, the p-values for the t-test and the Wilcoxon test are 0.46 and 0.11 , respectively. This example illustrates that in small sample sizes, the new adaptive procedure is able to detect a significant difference when both the t-test and the Wilcoxon test could not.

Typically, when the paired differences follow a normal distribution, the t-test is used; and when the normality assumption fails, the nonparametric Wilcoxon test is recommended. However, Freidlin, Miao and Gastwirth (2003) showed that this approach does not have high power when the paired differences are heavy-tailed. Randles and Hogg (1973) introduced an adaptive procedure that strictly maintains the size of the test and has high power when the distribution is heavy-tailed. Unfortunately, their procedure does not have high power for light to moderate tailed distributions. Freidlin et al. (2003) proposed an adaptive procedure (denoted by FMG) that uses the p-values of the Shapiro-Wilk normality test to choose an appropriate signed rank test to analyze the pairs. Their adaptive procedure has high power for a reasonable large class of moderate to heavy tailed distributions. However, their preliminary test is only asymptotically uncorrelated with the signed rank test used to analyze the paired sample, and the type I error for the procedure is slightly inflated. The FMG test was motivated by an environmental law case with sample size 16 and designed for 
relatively small samples. When the sample size is large, the Shapiro-Wilk and other tests of normality will detect minor deviations from normality. This may lead the FMG procedure to select a test that is good for a very heavy tailed distribution even if the differences are not that far from normal. The new method relying on a measure of "heavy-tailness" rather than the p-value of a preliminary test is applicable in large samples.

Mathematically, let $X_{1}, \ldots, X_{n}$ be i.i.d. paired differences from a continuous distribution $F$. The $X_{i}$ are naturally symmetric about a center $\mu$. We are interested in testing whether this center is equal to a known value $\mu_{0}$. Without loss of generality, assume that $\mu_{0}=0$, i.e. we are interested in testing:

$$
\begin{aligned}
& H_{0}: \mu=0 \\
& H_{a}: \mu \neq 0 .
\end{aligned}
$$

It's well known that when the $X_{i}$ s follow a normal distribution, the optimal test is the t-test (Lehmann, 1986). However, if we know that $X$ comes from a heavy-tailed distribution, e.g. $t_{2}$ or Cauchy, then the signed rank tests with $t_{2}$ (Gastwirth, 1970) or Cauchy scores (Capon, 1961) have higher power than the t-test. We propose an adaptive procedure (denoted by $\mathrm{MG}$ ) that first uses functions of order statistics of $\left|X_{i}\right|$ to obtain the information about the tailheaviness of the underlying distribution, and then use it to choose an appropriate signed rank test to analyze the pairs. The procedure strictly keeps the size of the test at the nominal level for all sample sizes, and has about the same power as the best signed rank test in a wide range of distributions, including the $\mathrm{t}$ family.

The paper is organized as follows: Section 2 introduces the preliminary test and its properties; Section 3 presents the percentiles of the preliminary test and how to use them to form the adaptive procedure. Power simulations for a variety of distributions are given in Section 4. The securities profit case data and the environmental data are analyzed in Section 5, using the new adaptive test and the results are compared with the paired t-test, Wilcoxon test and the FMG test.

\section{PROPERTIES OF THE PRELIMINARY TESTS}

\subsection{The preliminary tests}

We want an adaptive procedure to have high power over a wide range of distributions, from the light tailed uniform to the heavy-tailed Cauchy distributions. The tests used to analyze data at the second stage are signed rank tests. Those tests are based on the absolute ranks. It's well known (Lemma 8.3.11, Randles and Wolfe, 1979) that the ranks and the order statistics of the absolute values of the $X_{i}$ are independent. We choose functions of the ordered $\left|X_{i}\right|$ as the preliminary test, to measure the tail-heaviness. Doing so, the preliminary test and the tests used to analyze data are independent, which guarantees that the overall size of the adaptive procedure is kept at the nominal level.

The most commonly used measure of spread is the sample standard deviation, $s$. However, some other statistics, such as the median absolute deviation from the median are more robust measures of the spread. Under the null hypothesis of symmetric about 0 , the corresponding sample statistics are:

$$
\tilde{s}^{2}=\frac{1}{n} \sum_{i=1}^{n} X_{i}^{2} \quad \text { and } \quad \widetilde{M}=\frac{\operatorname{median}\left(\left|X_{1}\right|, \ldots,\left|X_{n}\right|\right)}{\Phi^{-1}(0.75)}
$$

where $\Phi(x)$ is the c.d.f. of standard normal. Note that the constant $1 / \Phi^{-1}(0.75)$ in $\tilde{M}$ is chosen to make $\tilde{M}$ a consistent estimator for the standard deviation when the underlying distribution is normal.

When the data are normally distributed, the ratio of two statistics, namely,

$$
s M=\frac{\tilde{s}}{\widetilde{M}}
$$

is near 1 . They will be greater (or less) than 1.0 when data has heavier (or lighter) tails than normal. The statistic $s M$ provides us indication of heaviness of the tails. We then use it as the preliminary test to choose the appropriate signed rank tests to analyze the paired data.

If one estimates the center by the sample median rather than the assumed 0 , the statistic $\tilde{M}$ becomes the median absolute deviation from the median, which is asymptotically equivalent to the semi-interquartile range, a robust estimate of spread (Hall and Welsh, 1985).

\subsection{Asymptotic properties of the preliminary test}

The following theorem shows that the statistic $s M$ is asymptotically normally distributed.

Theorem 2.1. Let $X$ be a symmetric continuous random variable with distribution function $F$. Let $f$ and $\sigma^{2}$ be its density function and the variance. Let $F(0)=\frac{1}{2}$ and $\xi=F^{-1}(0.75)$, i.e. 0 and $\xi$ are the median of $X$ and $|X|$, respectively. Let

$$
\begin{array}{rlrl}
\gamma_{1} & =\int_{0}^{\infty} x f(x) d x, & \gamma_{2}=\int_{0}^{\infty} x^{2} f(x) d x \\
\gamma_{3}=\int_{0}^{\infty} x^{3} f(x) d x, & \gamma_{4}=\int_{0}^{\infty} x^{4} f(x) d x .
\end{array}
$$

If the distribution $F$ satisfies the conditions $(A)-(C)$ stated in the Appendix, then statistic $s M$ is asymptotically normal. In other words,

$$
\sqrt{n}\left[s M-\frac{\sigma \Phi^{-1}(0.75)}{\xi}\right] \Rightarrow N\left(0, \sigma_{s M}^{2}\right)
$$


Table 1. Asymptotic means and standard deviations of the statistic sM

\begin{tabular}{llc}
\hline Distribution & Mean & Standard Deviation \\
\hline $\mathrm{U}(-1,1)$ & 0.7788 & 0.5225 \\
Normal & 1 & 0.9276 \\
Logistic & 1.1136 & 1.2181 \\
$0.95 N(0,1)+0.05 N\left(0,3^{2}\right)$ & 1.1388 & 1.5853 \\
$t_{5}$ & 1.1983 & 1.8542 \\
$0.9 N(0,1)+0.1 N\left(0,3^{2}\right)$ & 1.2406 & 1.7730 \\
Double Exponential & 1.3761 & 1.9388 \\
$0.9 N(0,1)+0.1 N\left(0,5^{2}\right)$ & 1.6741 & 3.3517 \\
\hline
\end{tabular}

where

$$
\begin{aligned}
\sigma_{s M}^{2}= & \Phi^{-2}(0.75) \\
& \times\left[\frac{2 \gamma_{4}-\sigma^{4}}{4 \xi^{2} \sigma^{2}}+\frac{\sigma^{2}}{16 \xi^{4} f^{2}(\xi)}+\frac{\int_{-\xi}^{\xi} x^{2} f(x) d x-\frac{1}{2} \sigma^{2}}{2 \xi^{3} f(\xi)}\right] .
\end{aligned}
$$

The proof of the Theorem is given in Appendix.

Table 1 lists the means and standard deviations of the asymptotic distributions of the $s M$ statistic for some commonly used symmetric distributions.

\section{THE ADAPTIVE PROCEDURES}

\subsection{The signed rank tests}

The signed rank tests used to analyze paired samples are expressed as:

$$
S=\sum_{i=1}^{n} a\left(R_{i}^{+}\right) \delta\left(X_{i}\right), \quad \text { with } a(i)=J\left(\frac{i}{n+1}\right)
$$

where $R_{i}^{+}$is the rank of $\left|X_{i}\right|$ among $\left|X_{1}\right|,\left|X_{2}\right|, \ldots,\left|X_{n}\right|$,

$$
\delta\left(X_{i}\right)=\left\{\begin{array}{ll}
1 & \text { if } X_{i}>0 \\
0 & \text { otherwise }
\end{array},\right.
$$

and $J$ is a score function appropriate for the data.

When the data comes from a light-tailed normal distributions or short-tailed uniform distributions, the normal scores test is known to have high power; when the tail-heaviness of the underlying distributions is somewhat medium, like logistic, double exponential or contaminated normal distributions, the Wilcoxon test is highly correlated with the maximin efficiency robust test (Gastwirth, 1966) and the Wilcoxon test should be used. Furthermore, if the data is heavy-tailed, e.g. from a $t_{2}$ or Cauchy distribution, then the appropriate signed rank test is the $t_{2}$ scores or the Cauchy scores test. We choose the following 4 score functions which include the extreme members of the $t$ family of distributions:

- $J_{1}(u)=\Phi^{-1}\left(\frac{1}{2}+\frac{1}{2} u\right)$, (Normal scores)

- $J_{2}(u)=u$, (Wilcoxon)

- $J_{3}(u)=\frac{3 \sqrt{2}}{2} u \sqrt{1-u^{2}},\left(t_{2}\right.$ scores $)$

- $J_{4}(u)=\frac{2 \tan \left(\frac{1}{2} \pi u\right)}{1+\tan ^{2}\left(\frac{1}{2} \pi u\right)}$. (Cauchy scores)

\subsection{Percentiles of the preliminary tests and adaptive procedures}

In order to form the cut-off of the adaptive procedure, we looked at both the empirical and asymptotic percentiles for statistic $s M$ for different distributions. Table 2 lists the empirical percentiles for statistic $s M$, for sample size 100. The distributions in the tables are arranged according to their tail heaviness, from the very heavy-tailed Cauchy to the light-tailed normal and uniform distributions. In the tables, C. Norm(5) (or C. Norm(3)) represents Tukey's $10 \%$ contaminated normal $0.9 N(0,1)+0.1 N\left(0,5^{2}\right)$ (or $0.9 N(0,1)+0.1 N\left(0,3^{2}\right)$ ). The results are based on $10^{5}$ simulations.

The general idea of the adaptive procedure is to use the sampling distribution of the preliminary test on a variety of underlying distributions to create cut-off values to classify the sample as light, medium, heavy or very heavy tailed. Then one chooses the signed rank test that is known to have high power for a distribution with tails of that type. We start with the heavy tailed Cauchy distribution, then move down the tail-heaviness to the light-tailed normal distribution, and finally to the short-tailed uniform distribution. Table 2 shows that more than $95 \%$ of Cauchy samples have $s M$ values bigger than 2.7 ( $5^{t h}$ percentile), and more than $80 \%$ of $t_{2}$ samples have $s M<2.7$. Thus 2.7 is chosen as the cut-off value of $s M$ between the Cauchy scores test and the $t_{2}$ scores test. In other words, if $s M \geq 2.7$, the Cauchy scores test is used. If $s M$ is slightly less than 2.7 , the $t_{2}$ scores test should be used. Furthermore, Table 2 indicates that more than $97.5 \% t_{2}$ samples, more than $90 \%$ contaminated normal samples $\left(0.9 N(0,1)+0.1 N\left(0,5^{2}\right)\right)$ and more than $80 \%$ double exponential samples have $s M$ values larger than 1.2 $\left(2.5^{s t}, 10^{t h}\right.$ and $20^{t h}$ percentiles, respectively), while about $80 \%$ of logistic samples have $s M$ values smaller than 1.2 $\left(80^{t h}\right.$ empirical percentile). Therefore we choose cut-off 1.2 between the $t_{2}$ scores test and the Wilcoxon test. This means that if $s M$ is between 1.2 and 2.7, the $t_{2}$ scores test is used. Similarly, we choose 1.02 as the cut-off between the Wilcoxon test and the normal scores test. In other words, if $s M$ is between 1.02 and 1.2 , the Wilcoxon test is used, and if $s M<1.02$, the normal scores test is used. The cut off 1.02 was chosen because for sample size 100 , all the uniform samples have $s M$ values smaller than 1 , but for sample size 25 , about $95 \%$ of uniform samples have $s M$ values less than 1.02. Furthermore, the Wilcoxon test has high power on both normal and logistic distributions. Consequently, the new adaptive procedure, denoted by MG, is the following:

the $M G$ Adaptive Test:

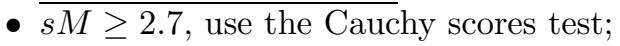

- $2.7>s M \geq 1.2$, use the $t_{2}$ scores test;

- $1.2>s M \geq 1.02$, use the Wilcoxon test;

- $s M<1.02$, use the Normal scores test. 
Table 2. Percentiles of the statistic $s M$ on a Variety of Distributions $(n=100)$

\begin{tabular}{rcccccccc}
\hline & Cauchy & $t_{2}$ & C. Norm(5) & D. Exp. & C. Norm(3) & Logistic & Normal & Uniform \\
\hline $1 \%$ & 2.0944 & 1.1450 & 1.0218 & 1.0165 & 0.9089 & 0.8827 & 0.8237 & 0.6834 \\
$2.5 \%$ & 2.3968 & 1.2135 & 1.0894 & 1.0587 & 0.9439 & 0.9106 & 0.8458 & 0.6951 \\
$5 \%$ & 2.7533 & 1.2804 & 1.1563 & 1.0974 & 0.9789 & 0.9365 & 0.8661 & 0.7062 \\
$10 \%$ & 3.2813 & 1.3696 & 1.2426 & 1.1461 & 1.0219 & 0.9688 & 0.8903 & 0.7197 \\
$20 \%$ & 4.1983 & 1.4984 & 1.3588 & 1.2110 & 1.0803 & 1.0107 & 0.9230 & 0.7377 \\
$25 \%$ & 4.6705 & 1.5564 & 1.4065 & 1.2372 & 1.1053 & 1.0278 & 0.9360 & 0.7450 \\
$30 \%$ & 5.1899 & 1.6129 & 1.4504 & 1.2614 & 1.1282 & 1.0434 & 0.9481 & 0.7517 \\
$40 \%$ & 6.3855 & 1.7309 & 1.5335 & 1.3079 & 1.1712 & 1.0736 & 0.9712 & 0.7646 \\
$50 \%$ & 7.9517 & 1.8615 & 1.6167 & 1.3540 & 1.2140 & 1.1020 & 0.9934 & 0.7772 \\
$60 \%$ & 10.219 & 2.0171 & 1.7036 & 1.4026 & 1.2607 & 1.1330 & 1.0169 & 0.7907 \\
$70 \%$ & 13.914 & 2.2263 & 1.8008 & 1.4583 & 1.3133 & 1.1676 & 1.0439 & 0.8057 \\
$75 \%$ & 16.798 & 2.3644 & 1.8554 & 1.4911 & 1.3439 & 1.1879 & 1.0591 & 0.8145 \\
$80 \%$ & 21.114 & 2.5508 & 1.9191 & 1.5284 & 1.3787 & 1.2106 & 1.0768 & 0.8246 \\
$90 \%$ & 43.597 & 3.2484 & 2.0970 & 1.6356 & 1.4768 & 1.2753 & 1.1265 & 0.8531 \\
$95 \%$ & 87.661 & 4.2296 & 2.2534 & 1.7303 & 1.5655 & 1.3336 & 1.1703 & 0.8790 \\
$97.5 \%$ & 173.56 & 5.6334 & 2.4012 & 1.8198 & 1.6458 & 1.3874 & 1.2116 & 0.9033 \\
$99 \%$ & 436.05 & 8.5566 & 2.5813 & 1.9310 & 1.7480 & 1.4548 & 1.2657 & 0.9340 \\
\hline
\end{tabular}

\section{SIZE AND POWER OF THE NEW ADAPTIVE PROCEDURE}

\subsection{Size}

The preliminary test $s M$ is a function of ordered absolute values of the differences, and the tests used to analyze data are the signed rank tests based on absolute ranks. For continuous distributions, the signed ranks and the absolute order statistics are independent (Lemma 8.3.11 of Randles and Wolfe, 1979). Hence the adaptive procedure $M G$ maintains the size at the nominal level (Lemma 11.6.3 of Randles and Wolfe, 1979). Our simulation results confirm that the adaptive test $M G$ keeps the size at its nominal level for sample sizes as small as 10, when the asymptotic normal distribution is used to calculate the p-value for the signed rank tests.

Freidlin, Miao and Gastwirth (2003) proposed an adaptive procedure (denoted by FMG test) for paired data, using the p-value of the Shapiro-Wilk test to choose appropriate signed rank tests. They proved that the Shapiro-Wilk test is asymptotically uncorrelated with the signed rank test. Simulations showed that their method had nearly nominal level in sample sizes 10-25. The adaptive test MG strictly maintains the size at its nominal level and is a slightly improvement over the FMG test in this regard.

In contrast to the situation under the null hypothesis, under the alternative hypothesis, the $s M$ statistic and the signed rank tests are somewhat correlated. Simulation studies, suggested by a referee, show that the magnitude of the correlations are usually less than 0.2 at meaningful alternatives. Furthermore, under the alternatives, the $s M$ statistic and the p-value of the chosen test are also related. Simulations show that for light-tailed (heavy-tailed) underlying distributions, smaller values of the $s M$ statistic are associated with smaller (larger) p-values. This is consistent with theory, as the expected values of the $s M$ statistic are small on lighttailed distributions and small values of the $s M$ lead to the selection of either the Normal scores test or the Wilcoxon test at the second stage. Those two tests are known to have high power for light-tailed normal and logistic distributions.

\subsection{Power}

The following graphs are the size and power comparisons for 3 different procedures: The adaptive procedure proposed by Freidlin, Miao and Gastwirth (2003), the optimal signed rank tests for the particular distribution (denoted by Best SRT) and the new MG adaptive procedure. For contaminated normal distributions $\left(0.9 N(0,1)+0.1 N\left(0,3^{2}\right)\right)$, the Wilcoxon test is considered to be highly efficient (Hodges and Lehmann, 1961), and for uniform distributions, the normal score test is taken to be the best signed rank test. The graphs are based on $10^{4}$ simulations for samples of size 100 .

Figures 1 and 2 indicate that the two adaptive procedures: FMG and MG, have about the same power as the best signed rank tests for normal and logistic distributions. It's interesting to note that for double exponential distributions (Figure 3) the two adaptive procedures MG and FMG have slightly higher power than the the best signed rank test (the sign test). For heavy-tailed $t_{2}$ and contaminated normal distributions $\left(0.9 N(0,1)+0.1 N\left(0,3^{2}\right)\right)$, the MG adaptive test has about the same power as the best signed rank test; their powers are slightly higher than the FMG procedure. For sample size 100, the power of the MG procedure is about 2-6\% higher than the FMG test for contaminated normal distributions. But when the sample size increases to 200, the power of the MG test is significantly higher than the FMG test (about 10-15\% higher). (Also see Table 3 below.) This occurs because in large samples, the Shapiro-Wilk test will yield a low p-value even when the 
Table 3. Power comparison for the FMG, MG and the Best SRT tests

\begin{tabular}{lccccc}
\hline Distribution & Sample Size & Alternative & MG & FMG & Best SRT \\
\hline Normal & 25 & 0.627 & 0.8268 & 0.8377 & 0.8460 \\
& 100 & 0.31 & 0.8565 & 0.8523 & 0.8700 \\
& 200 & 0.215 & 0.8415 & 0.8324 & 0.8497 \\
\hline Logistic & 25 & 1.11 & 0.8288 & 0.8487 & 0.8479 \\
& 100 & 0.545 & 0.8554 & 0.8629 & 0.8691 \\
& 200 & 0.374 & 0.8478 & 0.8379 & 0.8566 \\
\hline Double Exponential & 25 & 0.805 & 0.8375 & 0.8577 & 0.8496 \\
& 100 & 0.365 & 0.8733 & 0.8747 & 0.8558 \\
& 200 & 0.25 & 0.8700 & 0.8652 & 0.8478 \\
\hline$t_{2}$ & 25 & 0.878 & 0.8134 & 0.829 & 0.8497 \\
& 100 & 0.4 & 0.8432 & 0.8145 & 0.8524 \\
$0.9 N(0,1)+0.1 N\left(0,3^{2}\right)$ & 200 & 0.275 & 0.8369 & 0.7997 & 0.8427 \\
\hline & 100 & 0.735 & 0.8374 & 0.8563 & 0.8512 \\
& 200 & 0.35 & 0.8379 & 0.7703 & 0.8526 \\
& & 0.245 & 0.8316 & 0.6953 & 0.8557 \\
\hline
\end{tabular}

Power for Normal Distributions, $\mathrm{n}=100$

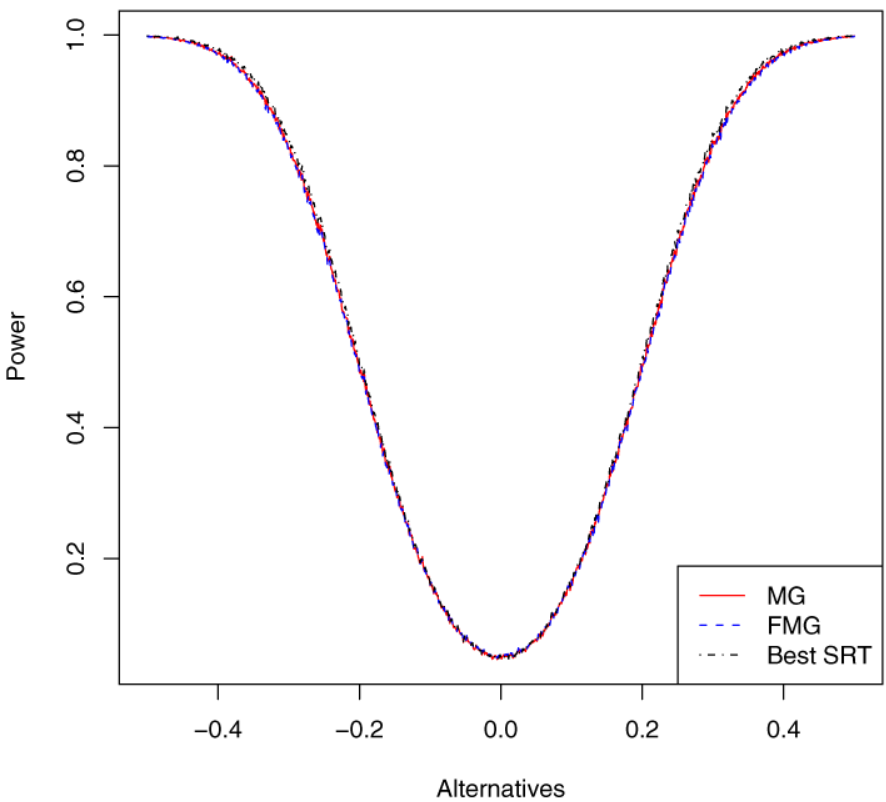

Figure 1. Power comparison for normal distributions.

data are nearly normal, e.g. contaminated normal, and the FMG procedure will choose the $t_{2}$ or Cauchy scores test too often on contaminated normal distributions. For uniform distributions, again the MG adaptive test has about the same power as the normal scores test, but the FMG adaptive procedure has significantly lower power compared to the other two procedures. This is not surprising as the normal scores test, which is known to have high power on uniform distributions, is not one of the second stage choices in the FMG test.

The following table presents the powers of the FMG, the
Power for Logistic Distributions, $n=100$

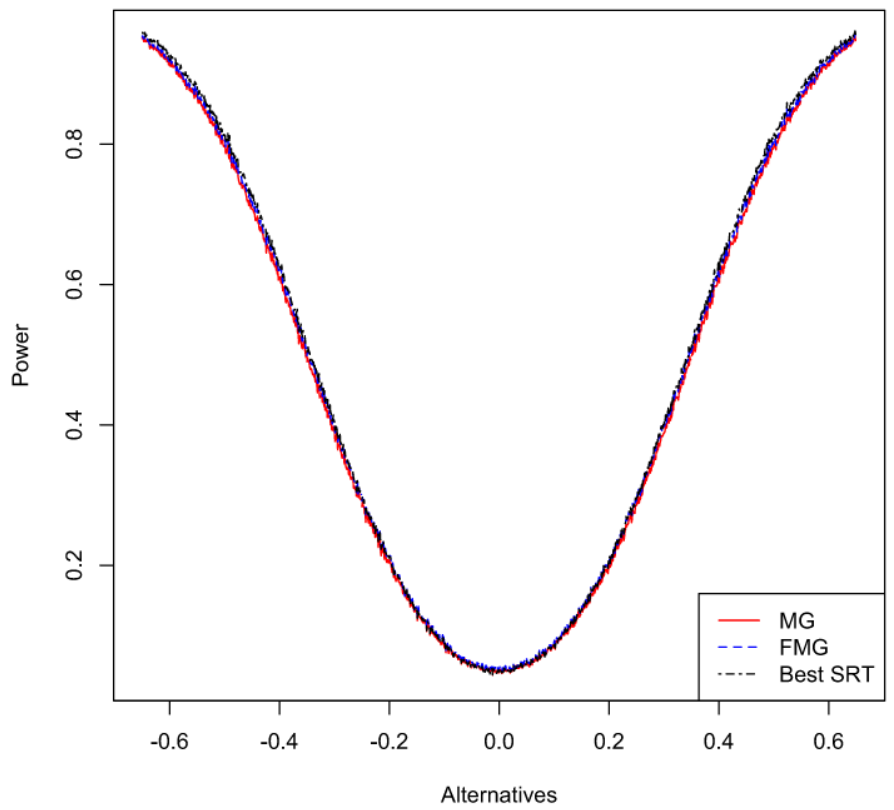

Figure 2. Power comparison for logistic distributions.

MG and the best signed rank test for sample sizes at 200, for Normal, Logistic, Double exponential, $t_{2}$ and contaminated Normal distributions. The powers of the tests for samples of size 25 and 100 are also included. The purpose of the table is to illuminate the roles of the sample sizes in determining the properties of the adaptive test. The alternatives are chosen so that the best signed rank test has about $85 \%$ power. The results are based on $10^{4}$ simulations. The table clearly shows that for $t_{2}$ and contaminated normal distributions, when the sample size gets larger, the new adaptive test $M G$ is better than the FMG test. 
Power for Double Exponential Distributions, $n=100$

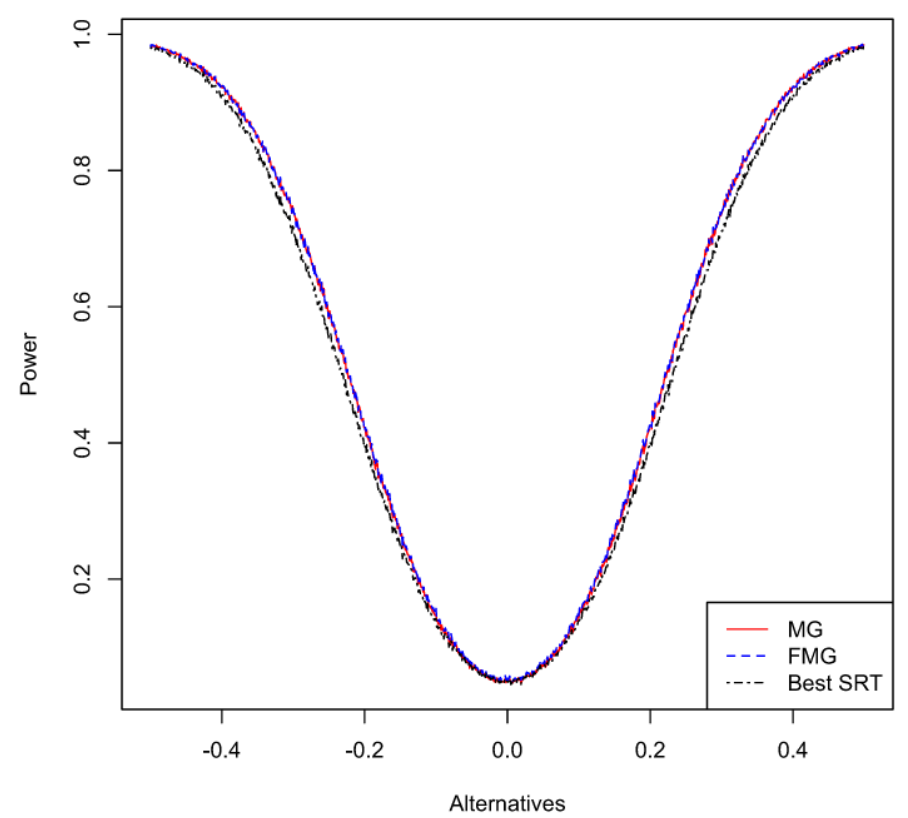

Figure 3. Power comparison for double exponential distributions.

Power for $\mathbf{t 2}$ Distributions, $\mathrm{n}=100$

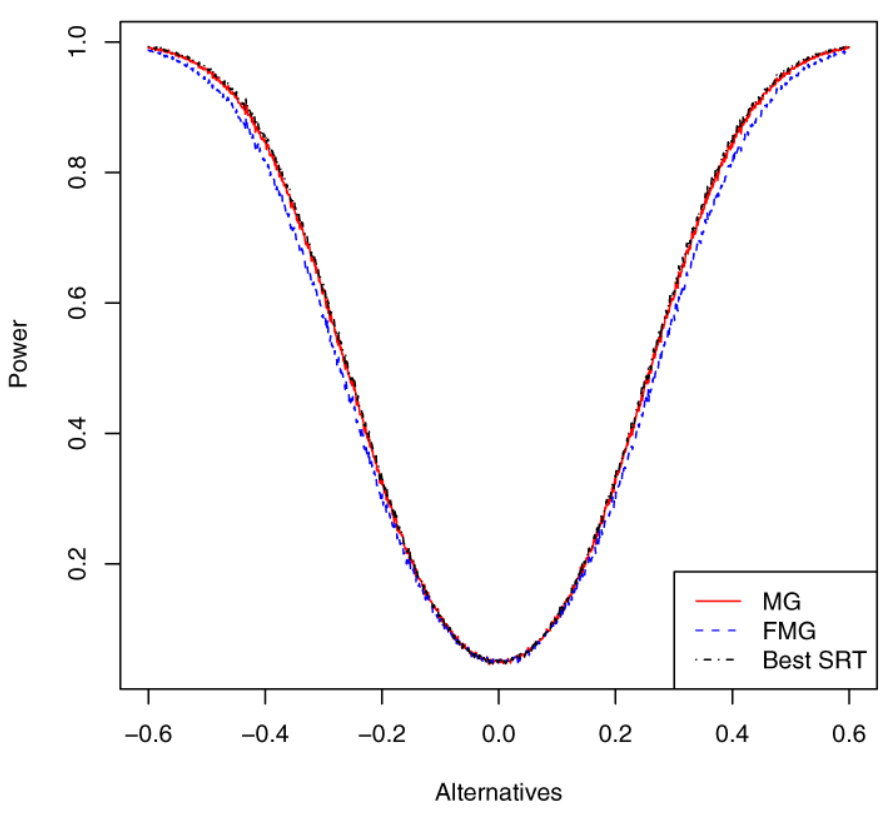

Figure 4. Power comparison for $t_{2}$ distributions.

Simulations were also performed on the Cauchy distribution for samples of size 25, 100 and 200. The powers of the FMG, the MG and the Cauchy scores tests are about the same.

The main reason that the adaptive procedure MG is pow-

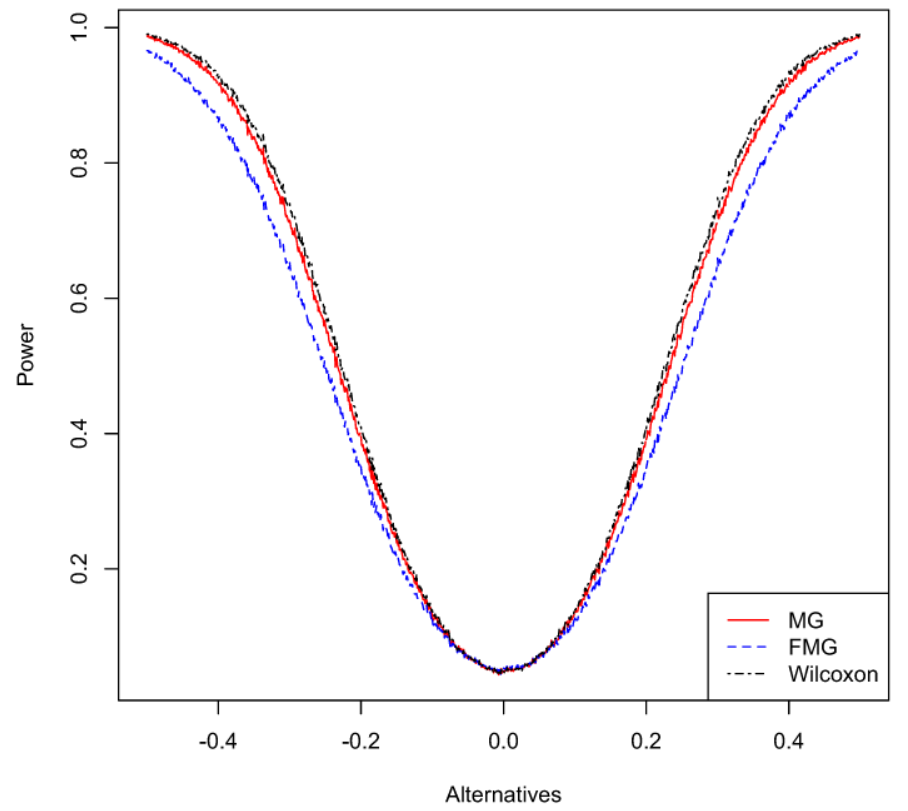

Figure 5. Power comparison for contaminated normal distributions $\left(0.9 N(0,1)+0.1 N\left(0,3^{2}\right)\right)$.

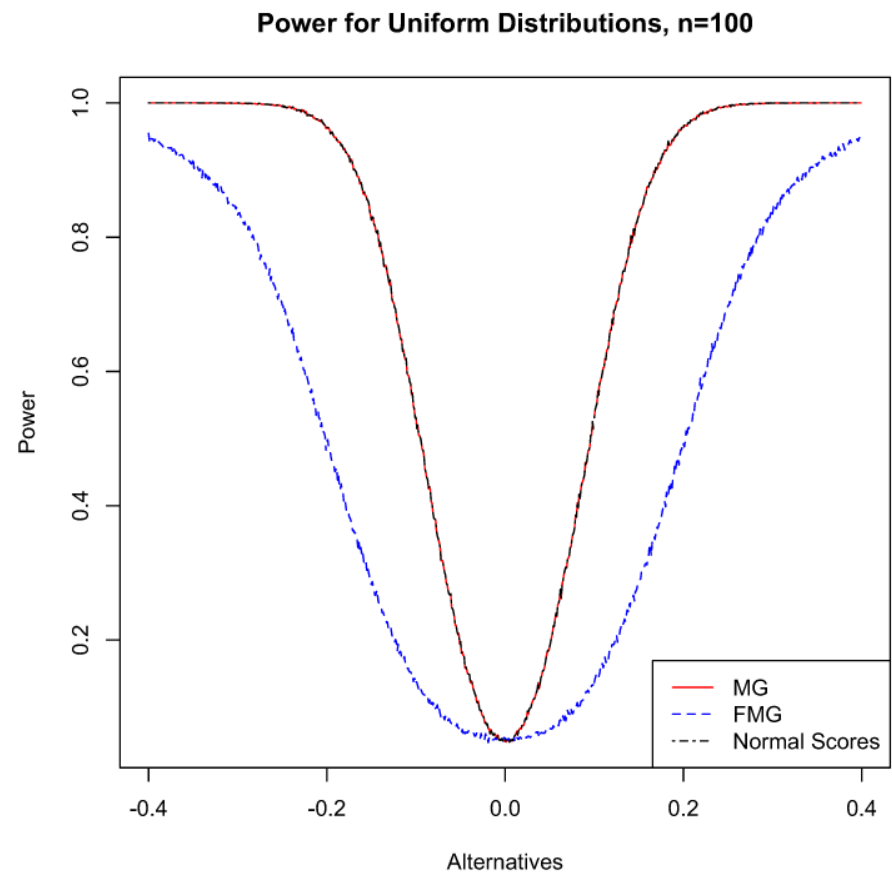

Figure 6. Power comparison for uniform distributions.

erful is that the preliminary test chooses either the best or the second best signed rank test most of the time. Simulations show that for normal distributions, our adaptive test chooses the normal scores test about $62 \%$ of the time, the Wilcoxon test about $35 \%$ of the time, and only chooses the 
$t_{2}$ scores test about $3 \%$ of the time. For samples from a $t_{2}$ distributions, the $M G$ procedure chooses the $t_{2}$ scores test about $82 \%$ of the time, the Cauchy scores test about $14 \%$ of time, and the Wilcoxon test about $4 \%$ of time.

These power simulations indicate that the adaptive procedure MG keeps the size at the nominal level and generally has the same power as the best signed rank test for a variety of symmetric distributions. This procedure is recommended for use in practice.

\section{EXAMPLES}

\subsection{The securities hypothetical profit data}

This data concerns the hypothetical profit estimates for 157 IPOs during an 18-month period. The p-value of paired t-test is 0.027 , indicating that the two measurements from two different regulators are statistically different. But the data is clearly not normal (the p-value of the Shapiro-Wilk test is $\left.1.4 * 10^{-15}\right)$. Applying the adaptive procedure, the preliminary test statistic $\mathrm{sM}=2.44$. Accordingly, the $\mathrm{MG}$ procedure chooses the $t_{2}$ scores test. The two-sided p-value for the $t_{2}$ scores test is 0.009 , noticeably smaller than 0.027 , the p-value of the paired test. In this example, the FMG adaptive test chooses Cauchy scores test, which yields a pvalue of 0.064 .

\subsection{The environmental data}

This data consists of 24 soil samples for lead analysis, 2 from each of the 12 different locations in the Detroit area. We are interested in whether the two measurements from the same location are the same. The p-value of the Shapiro-Wilk test is 0.029 , so the differences are not normally distributed. Applying the new adaptive procedure, the preliminary test statistic $s M$ is 2.07 . So, the new adaptive test chooses the $t_{2}$ scores test, which yields a p-value of 0.0377 , indicating that the two measurements are not the same.

In contrast to the above analysis, the p-value for the paired t-test is 0.46 , which is not even close to statistical significant. In this example, the p-value of the Wilcoxon test is 0.11 , much smaller than the 0.46 , but still not significant at the commonly accepted $5 \%$ level. The FMG procedure chooses the Wilcoxon test (based on the 0.029 p-value of the Shapiro-Wilk test) and does not detect the significant difference, but performs better than the paired t-test.

This example shows that for small heavy-tailed nonnormal samples, the new adaptive procedure MG is able to detect the significant difference while the paired t-test, the Wilcoxon test and the FMG adaptive test cannot.

\section{FINAL REMARKS}

In addition to the MG adaptive procedure, we also explored the adaptive test based on the ratio of $\tilde{s} / \tilde{J}$, where $\tilde{J}=\sqrt{\frac{\pi}{2}} \frac{1}{n} \sum_{i=1}^{n}\left|X_{i}\right|$, the mean absolute deviation from the known center 0. Combing the Central Limit Theorem and the delta-method, it can be shown that $\tilde{s} / \tilde{J}$ is also asymptotical normal. However, the asymptotical means and coefficient of variations of the $\tilde{s} / \tilde{J}$ are less spread out than the $s M=\tilde{s} / \tilde{M}$ for most commonly occurring symmetric distributions. This means that the statistic $\tilde{s} / \tilde{M}$ is better to distinguish different distributions. Power simulation also confirmed that the adaptive procedure based on $\tilde{s} / \tilde{M}$ has slightly higher power than the procedure based on the preliminary test $\tilde{s} / \tilde{J}$.

Studies showed that adaptive procedures for one-sample and two-sample problems work well over a wide range of distributions (Hogg 1974; Hogg, Fisher and Randles, 1975 and Hill, Padmanabhan and Puri, 1988). This article proposes an adaptive procedure for paired data. This adaptive test, MG, keeps its size at the nominal level and has nearly the same power as the best signed rank test for a wide range of distributions, including the family of $t$ distributions. It can be recommended to use in practice.

\section{ACKNOWLEDGEMENT}

The authors would like to thank reviewers for several helpful suggestions.

\section{APPENDIX}

The following are the conditions $(\mathrm{A})-(\mathrm{C})$ in Theorem 2.1:

(A) $F^{-2}(x)$ is continuous on the open unit interval $(0,1)$ and satisfies a first order Lipschitz condition on every interval bounded away from 0 and $1 ;\left(F^{-2}\right)^{\prime}(x)$ exists and is continuous except on a set of Jordan content 0 ;

(B) $f(\xi) \neq 0$ and the Riemann integral $\int_{0}^{1}\left(F^{-2}\right)^{\prime}\left(\frac{u+1}{2}\right)[u(1-u)]^{\frac{1}{2}} d u$ converges absolutely;

(C) For $i=1,2$ there exist $\delta_{i} \in(0,1)$ such that $\forall k_{i}>0$ $\exists M_{i}<\infty$ such that

(C1) if $0<u_{1}, u_{2}<\delta_{1}$ and $k_{1}^{-1}<u_{1} / u_{2}<k_{1}$, then

$$
M_{1}^{-1}<\frac{F^{-1}\left(\frac{u_{1}+1}{2}\right)\left(F^{-1}\right)^{\prime}\left(\frac{u_{1}+1}{2}\right)}{F^{-1}\left(\frac{u_{2}+1}{2}\right)\left(F^{-1}\right)^{\prime}\left(\frac{u_{2}+1}{2}\right)}<M_{1} ;
$$

(C2) if $1-\delta_{2}<u_{1}, u_{2}<1$ and $k_{2}^{-1}<\left(1-u_{1}\right) /\left(1-u_{2}\right)<$ $k_{2}$, then

$$
M_{2}^{-1}<\frac{F^{-1}\left(\frac{u_{1}+1}{2}\right)\left(F^{-1}\right)^{\prime}\left(\frac{u_{1}+1}{2}\right)}{F^{-1}\left(\frac{u_{2}+1}{2}\right)\left(F^{-1}\right)^{\prime}\left(\frac{u_{2}+1}{2}\right)}<M_{2} .
$$

Proof of Theorem 2.1. As $X$ is symmetric about 0 ,

$$
\begin{aligned}
& f_{|X|}(x)=2 f(x), \quad F_{|X|}(x)=2 F(x)-1, \quad \text { and } \\
& F_{|X|}^{-1}(u)=F^{-1}\left(\frac{u+1}{2}\right) .
\end{aligned}
$$


It can be shown that

$$
\begin{aligned}
E\left(\tilde{s}^{2}\right) & =\sigma^{2}, \quad E(\tilde{M})=\frac{\xi}{\Phi^{-1}(0.75)}, \\
\operatorname{Var}\left(\tilde{s}^{2}\right) & =\frac{1}{n}\left[2 \gamma_{4}-\sigma^{4}\right], \\
\operatorname{Var}(\tilde{M}) & =\frac{1}{16 n f^{2}(\xi)\left[\Phi^{-1}(0.75)\right]^{2}} .
\end{aligned}
$$

Let

$$
M=\text { median }\left(\left|X_{1}\right|, \ldots,\left|X_{n}\right|\right), \quad I_{i}= \begin{cases}1 & \text { if }\left|X_{i}\right|<\xi \\ 0 & \text { otherwise }\end{cases}
$$

Consider the interval $(\xi, M)$. Note that this interval contains $\frac{n}{2}-\sum I_{i}$ observations as $M$ is the $\frac{n+1}{2}$ th observation and $\sum_{i=1}^{n} I_{i}$ is the \# of observations less than $\xi$. Using the Bahadur representation, $f_{|X|}(\xi)(M-\xi)=2 f(\xi)(M-\xi) \approx$ $P(|X| \in(\xi, M))$. Consequently,

$$
2 n f(\xi)(M-\xi) \sim \frac{n}{2}-\sum I_{i}=-\sum_{i=1}^{n}\left(I_{i}-\frac{1}{2}\right) .
$$

This implies that

$$
M-\xi \sim-\frac{1}{2 n f(\xi)} \sum_{i=1}^{n}\left(I_{i}-\frac{1}{2}\right)
$$

Hence,

$$
\begin{aligned}
\operatorname{Cov}(\tilde{s}, M) & =\operatorname{Cov}\left(\frac{1}{n} \sum_{i=1}^{n} X_{i}^{2},-\frac{1}{2 n f(\xi)} \sum_{i=1}^{n}\left(I_{i}-\frac{1}{2}\right)\right) \\
& =-\frac{n}{2 n^{2} f(\xi)} \operatorname{Cov}\left(X_{1}^{2}, I_{1}-\frac{1}{2}\right) \\
& =-\frac{1}{2 n f(\xi)}\left[E\left(X^{2} I_{1}\right)-E\left(X_{1}^{2}\right) E\left(I_{1}\right)\right] \\
& =-\frac{1}{2 n f(\xi)}\left[\int_{-\xi}^{\xi} x^{2} f(x) d x-\frac{1}{2} \sigma^{2}\right]
\end{aligned}
$$

and

$$
\operatorname{Cov}(\tilde{s}, \tilde{M})=-\frac{1}{2 n f(\xi) \Phi^{-1}(0.75)}\left[\int_{-\xi}^{\xi} x^{2} f(x) d x-\frac{1}{2} \sigma^{2}\right] .
$$

Let $|X|_{(i)}$ be the order statistics of $\left|X_{i}\right|$. Since both $\tilde{s}^{2}$ and $\tilde{M}$ are L-statistics, they can be represented as:

$$
\frac{1}{n} \sum_{i=1}^{n} c_{i} h\left(|X|_{(i)}\right)+a|X|_{\left[\frac{n+1}{2}\right]}
$$

with appropriate function $h(\cdot)$ and constants $c_{i}$ and $a$, where $\left[\frac{n+1}{2}\right]$ is the largest integer smaller than $\frac{n+1}{2}$. The function $h$ and constants $c_{i}$ and $a$ corresponding to the statistics $\tilde{s}^{2}$ and $\tilde{M}$ are: (i) For $\tilde{s}^{2}, c=1, h(x)=x^{2}, a=0$;

(ii) For $\tilde{M}, c_{i}=0, h(x)=x^{2}, a=\Phi^{-1}(0.75)$.

The joint asymptotic normality of $\left(\tilde{s}^{2}, \tilde{M}\right)^{t}$ is obtained by applying Corollary 3 in Chernoff et al. (1967). It can be checked that the conditions $(\mathrm{A})-(\mathrm{C})$ listed in this Theorem are equivalent to the assumptions $A^{*}, B^{* *}$ and $E$ in Chernoff et al. (1967) with their function $H(u)=F^{-2}\left(\frac{u+1}{2}\right)$. According to Remark 9 of Chernoff et al. (1967), asymptotically $\left(\tilde{s}^{2}, \tilde{M}\right)^{t}$ is normal with mean $\left(\sigma^{2}, \frac{\xi}{\Phi^{-1}(0.75)}\right)^{t}$.

The Taylor expansion of $s M=\frac{\sqrt{\tilde{s}^{2}}}{\tilde{M}}$ at $\left(\sigma^{2}, \frac{\xi}{\Phi^{-1}(0.75)}\right)^{t}$ is:

$$
\begin{aligned}
s M= & \frac{\sqrt{\tilde{s}^{2}}}{\tilde{M}}=\frac{\sqrt{\sigma^{2}}}{\xi / \Phi^{-1}(0.75)}+\frac{1}{2 \sigma \xi / \Phi^{-1}(0.75)}\left(\tilde{s}^{2}-\sigma^{2}\right) \\
& -\frac{\sqrt{\sigma^{2}}}{\xi^{2} / \Phi^{-2}(0.75)}(\tilde{M}-\xi)+0_{p}\left(n^{-\frac{1}{2}}\right) \\
= & \frac{\sigma \Phi^{-1}(0.75)}{\xi}+\frac{\Phi^{-1}(0.75)}{2 \sigma \xi}\left(\tilde{s}^{2}-\sigma^{2}\right) \\
& -\frac{\sigma \Phi^{-2}(0.75)}{\xi^{2}}(\tilde{M}-\xi)+0_{p}\left(n^{-\frac{1}{2}}\right) .
\end{aligned}
$$

Consequently,

$$
\sqrt{n}\left[s M-\frac{\sigma \Phi^{-1}(0.75)}{\xi}\right] \Rightarrow N\left(0, \sigma_{s M}^{2}\right)
$$

with

$$
\begin{aligned}
\sigma_{s M}^{2}= & \frac{n \Phi^{-2}(0.75)}{4 \xi^{2} \sigma^{2}} \operatorname{Var}\left(\tilde{s}^{2}\right)+\frac{n \sigma^{2} \Phi^{-4}(0.75)}{\xi^{4}} \operatorname{Var}(\tilde{M}) \\
& -\frac{n \Phi^{-3}(0.75)}{\xi^{3}} \operatorname{Cov}\left(\tilde{s}^{2}, \tilde{M}\right) \\
= & \Phi^{-2}(0.75)\left[\frac{2 \gamma_{4}-\sigma^{4}}{4 \xi^{2} \sigma^{2}}\right. \\
& \left.+\frac{\sigma^{2}}{16 \xi^{4} f^{2}(\xi)}+\frac{\int_{-\xi}^{\xi} x^{2} f(x) d x-\frac{1}{2} \sigma^{2}}{2 \xi^{3} f(\xi)}\right] .
\end{aligned}
$$

\section{Received 11 November 2008}

\section{REFERENCES}

[1] Capon, J. (1961). Asymptotic Efficiency of Certain Locally Most Powerful Rank Tests. The Annals of Mathematical Statistics 32 88-100. MR0120739

[2] Chernoff, H., Gastwirth, J. L., and Johns Jr., M. V. (1967). Asymptotic distribution of linear combinations of functions of order statistics with applications to estimation. The Annals of Mathematical Statistics 38 352-372. MR0203874

[3] D'Agostino, R. B. and Cureton, E. E. (1973). A Class of Simple Linear Estimators of the Standard Deviation of the Normal Distribution. Journal of American Statistical Association 68 207-210.

[4] Weston Solutions of Michigan, INC. (2004). Phase I Summary Report for Detroit Lead Assessment Project, Great Lakes Smelting - 1640 East Euclid Street, Detroit, Wayne County, Michigan. 
[5] Freidlin, B., Miao, W., and Gastwirth, J. L. (2003). On the Use of the Shapiro-Wilk Test in Two-Stage Adaptive Inference for Paried Data from Moderate to Very Heavy Tailed Distributions. Biometrical Journal 45 887-900. MR2012347

[6] Gastwirth, J. L. (1966). On Robust Procedures. Journal of the American Statistical Association 61 929-948. MR0205397

[7] Gastwirth, J. L. (1970). On Robust Rank Tests. Nonparametric Techniques in Statistical Inference. Cambridge University Press, Cambridge. MR0277051

[8] Hall, P. and Welsh, H. (1985). Limit Theorems for the Median Deviation. Ann. Inst. Statist. Math. 37 27-36. MR0790373

[9] Hill, N. J., Padmanabhan, A. R., and Puri, M. L. (1988). Adaptive Nonparametric Procedures and Applications. Applied Statistics 37 205-218. MR0954744

[10] Hodges, J. L. and Lehmann, E. L. (1961). Comparison of the Normal Scores and Wilcoxon Test. Proc. Fourth Berkeley Sympo. Math. Stat. Prob. 1 307-317. MR0132645

[11] Hogg, R. V. (1972). More Light on the Kurtosis and Related Statistics. Journal of the American Statistical Association $\mathbf{6 7}$ 422-424.

[12] Hogg, R. V. (1974). Adaptive Robust Procedures: A Partial Review and Some Suggestions for Future Applications and Theory. Journal of the American Statistical Association 69 909-923. MR0461779

[13] Hogg, R. V., Fisher, D. M., and Randles, R. H. (1975). A Two-
Sample Adaptive Distribution-Free Test. Journal of the American Statistical Association $\mathbf{7 0}$ 656-661.

[14] Lehmann, E. L. (1986). Testing Statistical Hypothesis. Chapman \& Hall, New York.

[15] Randles, R. H. and Hogg, R. V. (1973). Adaptive Distribution Free Test. Communications in Statistics 2 337-356. MR0331628

[16] Randles, R. H. and Wolfe, D. A. (1979). Introduction to the Theory of Nonparametric Statistics. Krieger, Florida. MR0547836

[17] Sprent, P. and Smeeton, N. C. (2001). Applied Nonparametric Statistical Methods. Chapman \& Hall. MR2297847

\section{Weiwen Miao}

Department of Mathematics

Haverford College

Haverford, PA 19041.

E-mail address: wmiao@haverford.edu

\section{Joseph Gastwirth}

Department of Statistics

George Washington University

Washington, DC, 20052.

E-mail address: jlgast@gwu.edu 